\title{
DUAL PAIRS OF MATROIDS WITH COEFFICIENTS IN FINITARY FUZZY RINGS OF ARBITRARY RANK
}

\author{
Walter Wenzel ${ }^{1, *}$ \\ (Dedicated to Reinhard Diestel on the occasion of his $60^{\text {th }}$ birthday) \\ ${ }^{1}$ Universität Leipzig, Mathematisches Institut, Augustusplatz 10, 04109 Leipzig, Germany \\ Communicated by Günter Pilz \\ Original Research Paper \\ Received: Nov 15, 2019 - Accepted: Feb 11, 2020 \\ First published online: Mar 24, 2021 \\ () 2020 The Author(s)

\section{ABSTRACT} \\ Infinite matroids have been defined by Reinhard Diestel and coauthors in such a way that this class is (together with the finite \\ matroids) closed under dualization and taking minors. On the other hand, Andreas Dress introduced a theory of matroids \\ with coefficients in a fuzzy ring which is - from a combinatorial point of view - less general, because within this theory \\ every circuit has a finite intersection with every cocircuit. Within the present paper, we extend the theory of matroids with \\ coefficients to more general classes of matroids, if the underlying fuzzy ring has certain properties to be specified.
}

\section{KEYWORDS}

finite and infinite matroids, dual matroids, matroids with coefficients in a fuzzy ring, oriented matroids, valuated matroids

MATHEMATICS SUBJECT CLASSIFICATION (2020)

Primary 05B35; Secondary 05B25, 52B40, 52C40; 08A72, 51A25, 51D10, 51D20, 51D25

\section{INTRODUCTION}

For long time, matroids $M$ have only been examined when their groundset $E$ is finite, or, more generally, the rank of $M$ is finite, or, still more generally, $M$ is finitary; that means, all circuits of $M$ are finite. Concerning the classical theory of matroids, see for instance [11].

In 1966, R. Rado posed the question whether a theory about infinite matroids may be developed that satisfies at least the following two conditions:

These infinite matroids should contain the finitary matroids. Moreover, the class of infinite matroids (together with the finite matroids) should be closed under taking minors and duals. The last requirement fails in the class of finitary matroids.

A complete answer to Rado's question has been given in [4]. The authors did not only define infinite matroids satisfying the required conditions; they also find infinite versions for all the usual systems of axioms for matroids - in terms of independent sets, bases, the closure operator, circuits, and the rank function. Moreover, they prove that all of these systems of axioms are cryptomorphic.

\footnotetext{
* Corresponding author. E-mail: wenzel@math.uni-leipzig.de
} 
The question arises whether a similar theory may be developed for oriented matroids and related structures - such as, say, valuated matroids.

One cannot expect to get a theory with as many cryptomorphisms as for infinite matroids without any further structure. Particularly, the rank function does not control orientations - and infinite bases cannot be oriented in a similar matter as finite ones.

The question of how to define infinite oriented matroids - in terms of orientations of circuits and halfspaces induced by hyperplanes - will in the present paper be imbedded into a somewhat more general one:

A. W. M. Dress developed a theory of finite and infinite matroids with coefficients in a fuzzy ring in [6], which also satisfies the conditions posed by R. Rado, but is, at least from a combinatorial point of view, less general than the theory of infinite matroids as developed in [4]. More precisely, within this theory of matroids with coefficients, the intersection of a circuit and a cocircuit is - by the very definition - always finite, while there exist infinite matroids as defined in [4] that do not satisfy this property; such matroids are called wild matroids. A matroid is called a tame matroid if it is not a wild one. Concerning the existence of wild matroids, see [3].

Within [6] - as well as in several subsequent papers, see particularly [8] - all computations are tailored to tame matroids.

The present paper is dedicated to the problem whether some of these computations can be carried over to at least several classes of wild matroids. To this end, infinite sums in fuzzy rings have to be considered - without having any problems with convergence.

This is indeed unproblematic if the underlying fuzzy ring has the following property:

Every infinite sum of units is well determined by finitely many summands.

Such a fuzzy ring will be called a finitary one.

Particularly, the fuzzy ring $\mathbb{R} / / \mathbb{R}^{+}$corresponding to oriented matroids is finitary! This fact yields now a more or less canonical way to define infinite oriented matroids - as a special class of infinite matroids with coefficients in a finitary fuzzy ring.

Finite oriented matroids are introduced in [1] in terms of signed circuits; see also the papers [10] and [5], which are based on orientations of bases.

Valuated Matroids - of finite rank - are introduced in [9]. These can now also be defined even if the underlying matroid is wild, though the fuzzy ring corresponding to valuated matroids is not a finitary one. - It suffices that the above requirement concerning infinite sums of units does not necessarily hold generally but at least in special cases.

Technically, the paper is organized as follows:

In Section 2, we recall the concept of an infinite matroid as established in [4] and the definition of a fuzzy ring.

In Section 3, we introduce the concept of a (partial) finitary fuzzy ring and state several examples.

In Section 4, we present the general definition of a dual pair of matroids with coefficients in a fuzzy ring of arbitrary rank in terms of pairwise orthogonal circuit- and cocircuit functions which works in any case if this fuzzy ring is a finitary one. Moreover, we show that minors can be constructed arbitrarily, while dualization works - almost by definition - automatically.

Particularly, if $K$ is a field, it turns out - together with the results from [2] - that the dual pairs of matroids with coefficients in $K$ are precisely the dual pairs of tame thin sums matroids over $K$. More precisely, for a field $K$, these thin sums matroids are defined in terms of functions which allow infinite linear combinations over $K$ that are pointwise finite and, hence, well defined, while, in case of finitary fuzzy rings, any sum of units is automatically well defined.

In Section 5, we interpret cocircuit functions as hyperplane functions and derive several consequences concerning particular orthogonality relations.

This will particularly be examined in case of oriented matroids, weakly oriented matroids, and valuated matroids. 


\section{PRELIMINARIES}

In this section, we recall the concept of an infinite matroid as presented in [4], its dual, and the definition of a fuzzy ring, which up to now has served as a coefficient domain - particularly for matroids of finite rank.

If $M$ is an arbitrary set and $\mathfrak{M}$ is a set of subsets of $M$, then, $\mathfrak{M}_{\text {max }}$ will denote those sets in $\mathfrak{M}$ which are maximal with respect to inclusion. Note that $\mathfrak{M}_{\text {max }}$ may be empty even if $\mathfrak{M}$ is not empty.

DEFINITION 2.1. Suppose that $E$ is an arbitrary set and that $\mathfrak{T}$ is a set of subsets of $E$. Then the pair $M:=(E, \mathfrak{T})$ is a matroid defined on $E$ with $\mathfrak{T}$ as its independent sets if the following axioms hold:

(I1) $\varnothing \in \mathfrak{T}$.

(I2) If $I \in \mathfrak{T}$ and $J \subseteq I$, then one has $J \in \mathfrak{T}$; that means, $\mathfrak{T}$ is closed under taking subsets.

(I3) If $I \in \mathfrak{T} \backslash \mathfrak{T}_{\max }$ and $I^{\prime} \in \mathfrak{T}_{\max }$, then there exists some $x \in I^{\prime} \backslash I$ such that $I \cup\{x\} \in \mathfrak{T}$.

(I4) If $I \in \mathfrak{T}$ and $I \subseteq X \subseteq E$, then the set system $\left\{I^{\prime} \in \mathfrak{T} \mid I \subseteq I^{\prime} \subseteq X\right\}$ has a maximal element.

REMARK 2.2. Suppose that $M=(E, \mathfrak{T})$ is a matroid in the above sense. Then Axiom (I4) implies particularly:

$$
\mathcal{B}:=\mathfrak{T}_{\max } \neq \varnothing
$$

$\mathcal{B}$ is called the set of bases of $M$.

A matroid may also be defined in terms of its bases; see in [4, Theorem 4.1]. A set is independent if and only if it is contained in a base.

REMARK 2.3. Suppose that $M$ is a matroid defined on the set $E$ with $\mathcal{B}$ as its set of bases, and put

$$
\mathcal{B}^{*}:=\{E \backslash B \mid B \in \mathcal{B}\} .
$$

By [4, Theorem 3.1], $\mathcal{B}^{*}$ is again the set of bases of a matroid $M^{*}$, called the dual of $M$.

Trivially, one has $M^{* *}:=\left(M^{*}\right)^{*}=M$.

Next, we repeat the concepts of circuits, cocircuits, and hyperplanes of a matroid; see also [4].

DEFINITION 2.4. Assume that $M=(E, \mathfrak{T})$ is a matroid.

i) A subset $C$ of $E$ is called a circuit in $M$, if $C$ is a minimal dependent set; that means: $C \notin \mathfrak{T}$ but $J \in \mathfrak{T}$ for every proper subset $J$ of $C$.

ii) A subset $D$ of $E$ is a cocircuit in $M$, if $D$ is a circuit in the dual matroid $M^{*}$.

iii) A subset $H$ of $E$ is a hyperplane in $M$, if $H$ is a maximal set that does not contain a base. Equivalently, this means that $E \backslash H$ is a cocircuit in $M$.

At the end of this section, we repeat the concept of a fuzzy ring as defined in [6] as well as particular examples also stated in [6].

DEFINITION 2.5. A fuzzy ring $K=\left(K ;+; \cdot ; \epsilon ; K_{0}\right)$ consists of a set $K$, together with two binary operations $+, \cdot: K \times K \rightarrow K$, a specified element $\epsilon \in K$ and a specified subset $K_{0} \subseteq K$ such that the following axioms hold:

(FR0) $(K,+)$ and $(K, \cdot)$ are abelian semigroups with neutral elements 0 and 1 , respectively;

(FR1) $0 \cdot \kappa=0$ for all $\kappa \in K$;

(FR2) $\alpha \cdot\left(\kappa_{1}+\kappa_{2}\right)=\alpha \cdot \kappa_{1}+\alpha \cdot \kappa_{2}$ for all $\kappa_{1}, \kappa_{2} \in K$ and all $\alpha \in K^{*}:=\{\beta \in K \mid 1 \in \beta \cdot K\}$, the group of units in $K$;

(FR3) $\epsilon^{2}=1$;

(FR4) $K_{0}+K_{0} \subseteq K_{0}, K \cdot K_{0} \subseteq K_{0}, 0 \in K_{0}, 1 \notin K_{0}$;

(FR5) For $\alpha \in K^{*}$ one has $1+\alpha \in K_{0}$ if and only if $\alpha=\epsilon$;

(FR6) $\kappa_{1}, \kappa_{2}, \lambda_{1}, \lambda_{2} \in K$ and $\kappa_{1}+\lambda_{1}, \kappa_{2}+\lambda_{2} \in K_{0}$ implies $\kappa_{1} \cdot \kappa_{2}+\epsilon \cdot \lambda_{1} \cdot \lambda_{2} \in K_{0}$;

(FR7) $\kappa, \lambda, \kappa_{1}, \kappa_{2} \in K$ and $\kappa+\lambda \cdot\left(\kappa_{1}+\kappa_{2}\right) \in K_{0}$ implies $\kappa+\lambda \cdot \kappa_{1}+\lambda \cdot \kappa_{2} \in K_{0}$.

REMARK 2.6. Suppose that $K=\left(K ;+; ; \epsilon ; K_{0}\right)$ is a fuzzy ring.

i) (FR4),(FR5), and (FR7) imply $\kappa+\epsilon \cdot \kappa \in K_{0}$ for all $\kappa \in K$.

ii) (FR2) and (FR5) yield: (FR5') For $\alpha, \beta \in K^{*}$ one has $\alpha+\epsilon \cdot \beta \epsilon K_{0}$ if and only if $\alpha=\beta$. 
REMARK 2.7. The commutative unitary rings $R=(R ;+; \cdot)$ are (in a canonical correspondence to) exactly those fuzzy rings $K=\left(K ;+; \cdot ; \epsilon ; K_{0}\right)$ for which $K_{0}=\{0\}$. In this case, we have necessarily $\epsilon=-1$.

EXAMPLES 2.8. Suppose that $K=\left(K ;+; \cdot ; \epsilon ; K_{0}\right)$ is a fuzzy ring and that $U \leq K^{*}$ is a subgroup of the group of units in $K$. Then the quotient fuzzy ring $K / U$ is defined by

$$
K / U:=\left(\mathcal{P}(K)^{U} ;+; \cdot ; \epsilon \cdot U ; \mathcal{P}(K)_{0}^{U}\right),
$$

where

$$
\mathcal{P}(K)^{U}:=\{F \subseteq K \mid F \neq \varnothing, U \cdot F=F\}
$$

denotes the nonempty $U$-invariant subsets of $K$ and

$$
\mathcal{P}(K)_{0}^{U}:=\left\{F \in \mathcal{P}(K)^{U} \mid F \cap K_{0} \neq \varnothing\right\} .
$$

Sets in $\mathcal{P}(K)^{U}$ are added and multiplied as complexes.

$K / / U$ will denote the smallest fuzzy ring contained in $K / U$ that contains $(K / U)^{*} \cup\{\{0\}\}$.

If, particularly, $K=\mathbb{R}$ and $U=\mathbb{R}^{+}$equals the set of all real numbers or all positive real numbers, respectively, then we get with $L:=\mathbb{R} / / \mathbb{R}^{+}$:

$$
L^{*}=\left\{\mathbb{R}^{+}, \mathbb{R}^{-}\right\}, \quad L_{0}=\{\{0\}, \mathbb{R}\}, \quad L=L^{*} \dot{\cup} L_{0} .
$$

Moreover, we have the following addition and multiplication tables:

\begin{tabular}{c|llll}
+ & $\{0\}$ & $\mathbb{R}^{+}$ & $\mathbb{R}^{-}$ & $\mathbb{R}$ \\
\hline$\{0\}$ & $\{0\}$ & $\mathbb{R}^{+}$ & $\mathbb{R}^{-}$ & $\mathbb{R}$ \\
$\mathbb{R}^{+}$ & $\mathbb{R}^{+}$ & $\mathbb{R}^{+}$ & $\mathbb{R}$ & $\mathbb{R}$ \\
$\mathbb{R}^{-}$ & $\mathbb{R}^{-}$ & $\mathbb{R}$ & $\mathbb{R}^{-}$ & $\mathbb{R}$ \\
$\mathbb{R}$ & $\mathbb{R}$ & $\mathbb{R}$ & $\mathbb{R}$ & $\mathbb{R}$
\end{tabular}

\begin{tabular}{c|llll}
$\cdot$ & $\{0\}$ & $\mathbb{R}^{+}$ & $\mathbb{R}^{-}$ & $\mathbb{R}$ \\
\hline$\{0\}$ & $\{0\}$ & $\{0\}$ & $\{0\}$ & $\{0\}$ \\
$\mathbb{R}^{+}$ & $\{0\}$ & $\mathbb{R}^{+}$ & $\mathbb{R}^{-}$ & $\mathbb{R}$ \\
$\mathbb{R}^{-}$ & $\{0\}$ & $\mathbb{R}^{-}$ & $\mathbb{R}^{+}$ & $\mathbb{R}$ \\
$\mathbb{R}$ & $\{0\}$ & $\mathbb{R}$ & $\mathbb{R}$ & $\mathbb{R}$
\end{tabular}

\section{FINITARY FAMILIES OF UNITS AND FINITARY FUZZY RINGS}

In this section, we want to consider sums of infinitely many units in a fuzzy ring $K$ which may be well defined in a canonical manner. More precisely, we consider families $\left(x_{i}\right)_{i \in I}$ of elements in $K^{*} \cup\{0\}$; in what follows, such elements $x_{i}$ have to be added.

DEFINITION 3.1. Suppose that $K=\left(K ;+; \cdot ; \epsilon ; K_{0}\right)$ is a fuzzy ring.

i) Suppose that $\left(x_{i}\right)_{i \in I}$ is a family of elements in $K^{*} \cup\{0\}$. This family is called finitary, if the following finitary condition holds:

There exists a finite subset $I_{0} \subseteq I$ such that for all $j \in I \backslash I_{0}$ one has:

$$
\sum_{i \in I_{0}} x_{i}+x_{j}=\sum_{i \in I_{0}} x_{i}
$$

In other words, after having added all elements $x_{i}$ for $i \in I_{0}$, the sum does not change anymore by adding (finitely many) further elements $x_{j}$ for $j \in I \backslash I_{0}$.

In this case, we define the sum of the elements $x_{i}, i \in I$, as follows:

$$
\sum_{i \in I} x_{i}:=\sum_{i \in I_{0}} x_{i}
$$

ii) The fuzzy ring $K$ is called a finitary fuzzy ring, if every family of units in $K$ - and, hence, also every family of elements in $K^{*} \cup\{0\}-$ is finitary.

iii) The fuzzy ring $K$ is called a partial finitary fuzzy ring, if there exists at least one infinite family $\left(x_{i}\right)_{i \in I}$ of units in $K$ that is finitary. Note that this means that $I$ is infinite, while $K^{*}$ might be finite.

REMARK 3.2. Suppose that $K=\left(K ;+; ; \epsilon ; K_{0}\right)$ is a fuzzy ring.

i) Certainly, every finite family of units in $K$ is finitary:

Just put $I_{0}:=I$ in the above definition. 
ii) If $\left(x_{i}\right)_{i \in I}$ is a finitary family of elements in $K^{*} \cup\{0\}$, then their sum is well defined; that means, it does not depend on the finite subset $I_{0}$ of $I$ as specified:

Namely, suppose that $I_{1}, I_{2}$ are finite subsets of $I$ such that for both elements $k \in\{1,2\}$ and all $j \in I \backslash I_{k}$ one has

$$
\sum_{i \in I_{k}} x_{i}+x_{j}=\sum_{i \in I_{k}} x_{i}
$$

By applying this equation for $k=1$, induction on $\left|I_{2} \backslash I_{1}\right|$ yields:

$$
\sum_{i \in I_{1} \cup I_{2}} x_{i}=\sum_{i \in I_{1}} x_{i}
$$

By symmetry, the same equation holds by exchanging the roles of $I_{1}$ and $I_{2}$, which proves our claim.

EXAMPLES 3.3. $\quad$ i) There does not exist any unitary $\operatorname{ring} R$ that is partial finitary:

Namely, for every $r \in R$ and every $u \in R^{*}$ one has $r+u \neq r$.

ii) The fuzzy ring $\mathbb{R} / \mathbb{R}^{*}$ is a finitary fuzzy ring:

Every - finite - sum of at least 2 units equals $\mathbb{R}$.

iii) Also, the fuzzy ring $\mathbb{R} / / \mathbb{R}^{+}$corresponding to oriented matroids is a finitary one:

If $\left(x_{i}\right)_{i \in I}$ is a finite family of units all of which coincide, then there sum also takes this common value. If, otherwise, both of the units in $\mathbb{R} / / \mathbb{R}^{+}$are involved, then the sum all of these units equals $\mathbb{R}$.

EXAMPLE 3.4. If $\Gamma=(\Gamma, \cdot, \leq)$ is a linearly ordered abelian group, then the fuzzy ring $K_{\Gamma}$ that corresponds to valuated matroids with values in $\Gamma$ is determined by:

$$
K_{\Gamma}=\{0\} \dot{\cup} \Gamma \dot{\cup} \bar{\Gamma}, \quad K_{\Gamma}^{*}=\Gamma, \quad\left(K_{\Gamma}\right)_{0}=\{0\} \dot{\cup} \bar{\Gamma},
$$

where $\bar{\Gamma}$ is a disjoint copy of $\Gamma$, and 0 is a specified element neither contained in $\Gamma$ nor in $\bar{\Gamma}$.

Furthermore, we get the following addition and multiplication table, where $\alpha, \beta \in \Gamma$ are arbitrary but $\alpha<\beta$ in case of addition:

\begin{tabular}{c|ccccc}
+ & 0 & $\alpha$ & $\beta$ & $\bar{\alpha}$ & $\bar{\beta}$ \\
\hline 0 & 0 & $\alpha$ & $\beta$ & $\bar{\alpha}$ & $\bar{\beta}$ \\
$\alpha$ & $\alpha$ & $\bar{\alpha}$ & $\beta$ & $\bar{\alpha}$ & $\bar{\beta}$ \\
$\beta$ & $\beta$ & $\beta$ & $\bar{\beta}$ & $\beta$ & $\bar{\beta}$ \\
$\bar{\alpha}$ & $\bar{\alpha}$ & $\bar{\alpha}$ & $\beta$ & $\bar{\alpha}$ & $\bar{\beta}$ \\
$\bar{\beta}$ & $\bar{\beta}$ & $\bar{\beta}$ & $\bar{\beta}$ & $\bar{\beta}$ & $\bar{\beta}$
\end{tabular}

\begin{tabular}{l|lll}
$\cdot$ & 0 & $\beta$ & $\bar{\beta}$ \\
\hline 0 & 0 & 0 & 0 \\
$\alpha$ & 0 & $\alpha \cdot \beta$ & $\frac{\alpha \cdot \beta}{\alpha \cdot \beta}$ \\
$\bar{\alpha}$ & 0 & $\frac{\alpha \cdot \beta}{\alpha \cdot \beta}$
\end{tabular}

This fuzzy ring $K_{\Gamma}$ is a partial finitary one but not finitary:

A family of units $\left(\gamma_{i}\right)_{i \in I}$ in $K_{\Gamma}{ }^{*}=\Gamma$ is a finitary one if and only if this family takes a maximum value $\gamma_{i_{0}}$. In this case, we have:

$$
\sum_{i \in I} \gamma_{i}=\gamma_{i_{0}} \in \Gamma=K_{\Gamma}^{*}
$$

if the maximum value is attained exactly once. If, otherwise, the maximum value is attained at least twice, then we get

$$
\sum_{i \in I} \gamma_{i}=\overline{\gamma_{i_{0}}} \in\left(K_{\Gamma}\right)_{0}
$$

Before stating another class for finitary fuzzy rings, we fix the following

CONVENTION 3.5. Suppose that $\mathrm{F}$ is a field and that $U$ is a subgroup of $\mathbb{F}^{*}$. For $x \in \mathbb{F}^{*}$ and $n \in \mathbb{N}$, define $n(x \cdot U)$ recursively as follows:

$$
1(x \cdot U):=x \cdot U, \quad(n+1)(x \cdot U):=n(x \cdot U)+x \cdot U .
$$

PROPOSITION 3.6. Suppose that $\mathbb{F}$ is a finite field and that $U$ is a subgroup of $\mathbb{F}^{*}$ with at least 2 elements. Then the quotient structure $K:=\mathbb{F} / U$ is a finitary fuzzy ring. More precisely, we get for $q:=|\mathbb{F}|$ : 
i) One has

and, hence, also

$$
0 \in q(U), \quad 0 \in(q-1)(U)
$$

$$
0 \in n(U) \text { for all } n \geq(q-1) \cdot(q-2) \text {. }
$$

ii) For every infinite family $\left(x_{i}\right)_{i \in I}$ of units in $\mathbb{F}^{*}$, there exists a finite subfamily $\left(x_{i}\right)_{i \in I_{0}}$ such that for all $j \in I \backslash I_{0}$ one has:

$$
\sum_{i \in I_{0}} x_{i} \cdot U+x_{j} \cdot U=\sum_{i \in I_{0}} x_{i} \cdot U
$$

Proof. $\quad$ i) Clearly, we have $0 \in q(U)$, because $q$ is a power of $\operatorname{char}(\mathbb{F})$.

Furthermore, by assumption, there exists some $a \in U \backslash\{1\}$. Then we have $a^{q-1}=1$, and, hence

$$
0=(a-1)^{-1} \cdot\left(a^{q-1}-1\right)=\sum_{j=0}^{q-2} a^{j} \in(q-1)(U) .
$$

The last assertion in i) follows now from the following fact: $(q-1) \cdot(q-2)-1$ is the largest natural number $g$ that does not admit a presentation as follows:

$$
g=a \cdot(q-1)+b \cdot q \text { for appropriate } a, b \in \mathbb{N}_{0} .
$$

ii) Since $\mathbb{F}$ and, hence, also $\mathbb{F}^{*} / U$ are finite, the subgroup $U$ has only finitely many cosets in $\mathbb{F}^{*}$. Hence, it suffices to prove:

There exists some $N \in \mathbb{N}$ such that for all $n \in \mathbb{N}$ one has:

$$
N(U)=(N+n)(U) \text {. }
$$

If this is proved, then we obtain also

$$
N(x \cdot U)=(N+n)(x \cdot U) \text { for all } x \in \mathbb{F}^{*} \text { and all } n \in \mathbb{N} \text {. }
$$

Since $\mathbb{F}$ is finite, there exists some $M \in \mathbb{N}$ such that for all $n \in \mathbb{N}$ one has $|n(U)| \leq|M(U)|$. Furthermore, i) yields for all $n \in \mathbb{N}$ with $n \geq(q-1) \cdot(q-2)$ :

$$
M(U) \subseteq M(U)+n(U)=(M+n)(U) .
$$

By our choice of $M$, this means:

$$
M(U)=(M+n)(U) \text { for all } n \geq(q-1) \cdot(q-2) .
$$

Hence, the assertion follows for $N:=M+(q-1) \cdot(q-2)$.

REMARK 3.7. Since the relation $n(U)=m(U)$ implies $(n+1)(U)=(m+1)(U)$, too, we can even choose $N=M$ at the end of the last proof.

REMARK 3.8. In general, Proposition 3.6 does not hold for infinite fields:

Assume, for example, that $R$ is a valuation ring (with quotient field $\mathrm{F}$ ) whose maximal ideal $\mathfrak{m}$ satisfies the relation $[R: \mathfrak{m}]=2$, and put $U:=R^{*}=R \backslash \mathfrak{m}$. Then for all odd $n \in \mathbb{N}$ one has $n(U)=U$, while all even $n \in \mathbb{N}$ satisfy $n(U)=\mathfrak{m}$.

\section{DUAL PAIRS OF MATROIDS WITH COEFFICIENTS}

DEFINITION 4.1. Suppose that $K$ is an arbitrary fuzzy ring and that $M$ is a matroid defined on an arbitrary set $E$ with $\mathcal{C}$ as its set of circuits and $\mathcal{C}^{*}$ as its set of cocircuits.

A Dual Pair of matroids with coefficients in $K$ - and underlying matroid $M$ - consists of a family $\left(f_{C}\right)_{C \in \mathcal{C}}$ of circuit functions $f_{C}: E \rightarrow K^{*} \cup\{0\}$ and a family $\left(r_{D}\right)_{D \in C^{*}}$ of cocircuit functions $r_{D}: E \rightarrow$ $K^{*} \cup\{0\}$ such that the following properties hold:

(DPi) For all $C \in \mathcal{C}$ one has: $f_{C}^{-1}\left(K^{*}\right)=C$; for all $D \in \mathcal{C}^{*}$ one has: $r_{D}^{-1}\left(K^{*}\right)=D$.

(DPii) For all $C \in \mathcal{C}$ and all $D \in \mathcal{C}^{*}$, the following holds:

The family $\left(f_{C}(e) \cdot r_{D}(e)\right)_{e \in E}$ - of elements in $K^{*} \cup\{0\}$ - is a finitary family, and one has:

$$
\sum_{e \in E} f_{C}(e) \cdot r_{D}(e) \in K_{0}
$$


Moreover, for given units $\alpha_{C}, \beta_{D} \in K^{*}$, we say that two families $\left(f_{C}\right)_{C \in C}$ and $\left(r_{D}\right)_{D \in C^{*}}$ as in $(D P i)$ and (DPii) define the same Dual Pair of matroids with coefficients in $K$ as the families $\left(\alpha_{C} \cdot f_{C}\right)_{C \in \mathcal{C}}$ and $\left(\beta_{D} \cdot r_{D}\right)_{D \in \mathcal{C}^{*}}$.

REMARK 4.2. Definition 4.1 means that each circuit function and each cocircuit function is defined only up to some unit - as this has also been achieved in [6] and [8].

Moreover, it is trivial that this definition is selfdual in the following sense:

If $M$ is the underlying matroid of a dual pair of matroids with coefficients, then the same holds for $M^{*}$; just exchange the roles of circuit functions and cocircuit functions. However, note that the underlying matroid of a dual pair is uniquely determined if the roles of circuit functions on the one handside and of cocircuit functions on the other handside are specified.

REMARK 4.3. The matroid $M$ is called tame, if the intersection $C \cap D$ is finite for any circuit $C$ and any cocircuit $D$ in $M$; otherwise, $M$ is called a wild matroid. Hence, under assumption of (DPi), the matroid $M$ is tame if and only if the family $\left(f_{C}(e) \cdot r_{D}(e)\right)_{e \in E}$ exhibits only finitely many nonvanishing products - for any circuit $C$ and any cocircuit $D$. Moreover, this implies that the sum in (DPii) is automatically well defined. - Furthermore, if $K$ is a field, then in [2, Theorem 6.3] it is shown that the class of dual pairs of matroids with coefficients in $K$ is precisely the class of - dual pairs - of tame thin sums matroids over $K$. Note that for a field $K$, axiom (DPii) can hold only if $M$ is a tame matroid.

If, on the other handside, the fuzzy ring $K$ is finitary, then the sum in (DPii) is always well defined - even for any wild matroid $M$.

REMARK 4.4. Every matroid is the underlying matroid of a dual pair of matroids with coefficients in the universal fuzzy ring $K=\mathbb{R} / \mathbb{R}^{*}$ :

By [4, Lemma 3.1], a circuit and a cocircuit never meet in a singleton set. Hence, the sum in (DPii) either exhibits only products whose value is 0 or at least 2 nonzero products. In the last case, the sum equals $\mathbb{R}$ - which lies in $K_{0}$.

Next, we want to claim that, together with $M$, also every minor of $M$ is the underlying matroid of a dual pair of matroids with coefficients.

First, we recall the following definition, see in [4, Chapter 3].

DEFINITION 4.5. Suppose that $M=(E, \mathfrak{T})$ is a matroid - with $\mathfrak{T}$ as its system of independent sets and that $X \subseteq E$.

i) The restriction $M \mid X$ of $M$ to $X$ is defined by

$$
M \mid X:=(X, \mathfrak{T} \cap \mathcal{P}(X)) .
$$

In other words, the independent subsets in $M \mid X$ are the independent sets in $M$ which are contained in $X$.

ii) The contraction M.X of $M$ to $X$ is defined by

$$
M . X:=\left(M^{*} \mid X\right)^{*} .
$$

iii) A minor of $M$ is any matroid obtained from $M$ by a finite sequence of restrictions and contractions.

Now we can prove the following

PROPOSITION 4.6. Assume that $M=(E, \mathfrak{T})$ is the underlying matroid of a dual pair of matroids with coefficients in the fuzzy ring $K$. Then every minor of $M$ is also the underlying matroid of a dual pair of matroids with coefficients in $K$.

Proof. By Remark 4.2, it suffices to consider minors of the shape $M \mid X$ for $X \subseteq E$. By [4, Chapter 3], the circuits in $M^{\prime}:=M \mid X$ are those circuits $C$ in $M$ that are contained in $X$, while the cocircuits are the minimal nonempty sets of the shape $D^{\prime}=D \cap X$, where $D$ runs through the cocircuits of $M$.

Next, we define circuit functions and cocircuit functions in $M^{\prime}$ by continuing with the notations as in Definition 4.1. 
For any circuit $C$ in $M^{\prime}$, define the circuit function $f_{C}^{\prime}$ by $f_{C}^{\prime}(e):=f_{C}(e)$ for all $e \in X$. For any cocircuit $D^{\prime}$ in $M^{\prime}$, proceed as follows:

Choose a cocircuit $D$ in $M$ with $D^{\prime}=D \cap X$, and define the cocircuit function $r_{D^{\prime}}^{\prime}$ by $r_{D^{\prime}}^{\prime}(e):=r_{D}(e)$ for all $e \in X$. - Note that this function may depend not only on $D^{\prime}$ but also on the special choice of $D$; however, that is unimportant within this proof.

In any case, we get for $C, D^{\prime}, D$ as just considered:

$$
\sum_{e \in X} f_{C}^{\prime}(e) \cdot r_{D^{\prime}}^{\prime}(e)=\sum_{e \in E} f_{C}(e) \cdot r_{D}(e) \in K_{0}
$$

because $f_{C}(e)=0$ holds for every $e \in E \backslash X$. This proves what we want.

In what follows, assume that $K, M,\left(f_{C}\right)_{C \in \mathcal{C}},\left(r_{D}\right)_{D \in \mathcal{C}^{*}}$ are as in Definition 4.1.

REMARK 4.7. The condition that all families $\left(f_{C}(e) \cdot r_{D}(e)\right)_{e \in E}$ for circuits $C \in \mathcal{C}$ and cocircuits $D \in \mathcal{C}^{*}$ are finitary is fulfilled automatically if at least one of the following conditions i) or ii) holds:

i) The matroid $M$ is a tame matroid; see Remark 4.3. Tame matroids recover matroids with coefficients in a fuzzy ring as studied in [6].

ii) The fuzzy ring $K$ is finitary.

Furthermore, we want to point out the following

REMARK 4.8. In [3, Theorem 6.4] it is proved:

The class of tame thin sums matroids over a given field is closed under duality and under taking minors. Concerning the methods, analogous relations as in (DPii) are derived - where now $K_{0}=\{0\}$.

\section{HYPERPLANE FUNCTIONS}

We continue with the notations as in Definition 4.1.

REMARK 5.1. In what follows, we usually do not study directly cocircuit functions $r_{D}$ for $D \in \mathcal{C}^{*}$ but the corresponding hyperplane functions $s_{H}:=r_{E \backslash H}$ for $H \in \mathcal{H}$, where $\mathcal{H}$ denotes the set of hyperplanes in $M$. Then the last requirement in (DPii) means:

$$
\sum_{e \in E} f_{C}(e) \cdot s_{H}(e) \in K_{0}
$$

As a very important special case of Remark 5.1, we point out the following

LEMMA 5.2. Suppose that $C$ is a circuit in $M$ and that $H$ is a hyperplane in $M$ such that $|C \backslash H|=2$, say $C \backslash H=\{a, b\}$. Then we have:

i) $f_{C}(a) \cdot s_{H}(a)+f_{C}(b) \cdot s_{H}(b) \in K_{0}$.

ii) $f_{C}(a) \cdot s_{H}(a)=\epsilon \cdot f_{C}(b) \cdot s_{H}(b)$.

iii) $f_{C}(a) \cdot f_{C}(b)^{-1}=\epsilon \cdot s_{H}(b) \cdot s_{H}(a)^{-1}$.

Proof. In view of (DPi), i) is now a special case of Remark 5.1.

ii) follows directly from i) and Remark 2.6 ii).

iii) follows trivially from ii).

DEFINITION 5.3. A subset $L$ of $E$ is called a hyperline in $M$, if there exists a hyperplane $H$ in $M$ with $L \subseteq H \subseteq E$ such that $L$ is a hyperplane in the restriction $M \mid H$.

LEMMA 5.4. Suppose that $L$ is a hyperline in $M$ and that $H_{1}, H_{2}, H_{3}$ are pairwise distinct hyperplanes in $M$ containing $L$. Furthermore, suppose that $a_{i} \in H_{i} \backslash L$ for $1 \leq i \leq 3$. Then one has:

i) There exists a circuit $C$ in $M$ such that

$$
\left\{a_{1}, a_{2}, a_{3}\right\} \subseteq C \subseteq L \cup\left\{a_{1}, a_{2}, a_{3}\right\} .
$$

ii) One has:

$$
s_{H_{1}}\left(a_{2}\right) \cdot s_{H_{1}}\left(a_{3}\right)^{-1} \cdot s_{H_{2}}\left(a_{3}\right) \cdot s_{H_{2}}\left(a_{1}\right)^{-1} \cdot s_{H_{3}}\left(a_{1}\right) \cdot s_{H_{3}}\left(a_{2}\right)^{-1}=\epsilon .
$$

iii) If, moreover, $a_{1}^{\prime} \in H_{1} \backslash L$, then one has:

$$
s_{H_{3}}\left(a_{1}\right) \cdot s_{H_{2}}\left(a_{1}\right)^{-1}=s_{H_{3}}\left(a_{1}^{\prime}\right) \cdot s_{H_{2}}\left(a_{1}^{\prime}\right)^{-1} .
$$


Proof. i) Suppose that $U$ is a base of the restriction $M \mid L$. Then any set $U \cup\left\{a_{i}\right\}, 1 \leq i \leq 3$, is a base of $M \mid H_{i}$, and the sets $U \cup\left\{a_{1}, a_{2}\right\}, U \cup\left\{a_{1}, a_{3}\right\}, U \cup\left\{a_{2}, a_{3}\right\}$ are bases of $M$. This means that $D:=U \cup\left\{a_{1}, a_{2}, a_{3}\right\}$ is a dependent subset of $M$. By [4, Lemma 3.8], this means that $D$ contains a circuit $C$ that necessarily contains $\left\{a_{1}, a_{2}, a_{3}\right\}$.

ii) We apply Lemma 5.2 ii) to the three pairs $\left(C, H_{i}\right)$ for $1 \leq i \leq 3$ and obtain in view of $C \backslash H_{i}=\left\{a_{j}, a_{k}\right\}$ whenever $\{i, j, k\}=\{1,2,3\}$ :

$$
\begin{aligned}
& f_{C}\left(a_{2}\right) \cdot s_{H_{1}}\left(a_{2}\right)=\epsilon \cdot f_{C}\left(a_{3}\right) \cdot s_{H_{1}}\left(a_{3}\right), \\
& f_{C}\left(a_{3}\right) \cdot s_{H_{2}}\left(a_{3}\right)=\epsilon \cdot f_{C}\left(a_{1}\right) \cdot s_{H_{2}}\left(a_{1}\right), \\
& f_{C}\left(a_{1}\right) \cdot s_{H_{3}}\left(a_{1}\right)=\epsilon \cdot f_{C}\left(a_{2}\right) \cdot s_{H_{3}}\left(a_{2}\right) .
\end{aligned}
$$

By multiplying these three equations and shortening the three factors $f_{C}\left(a_{i}\right), 1 \leq i \leq 3$, we get:

$$
s_{H_{1}}\left(a_{2}\right) \cdot s_{H_{2}}\left(a_{3}\right) \cdot s_{H_{3}}\left(a_{1}\right)=\epsilon \cdot s_{H_{1}}\left(a_{3}\right) \cdot s_{H_{2}}\left(a_{1}\right) \cdot s_{H_{3}}\left(a_{2}\right) .
$$

This implies ii) directly.

iii) follows by applying ii) twice - once for $a_{1}^{\prime}$ instead of $a_{1}$.

PROPOSITION 5.5. Suppose that $L, H_{1}, H_{2}, H_{3}, a_{1}, a_{2}, a_{3}$ - and $C$ are as in Lemma 5.4. Then we get for all $e \in E$ :

$$
s_{H_{1}}\left(a_{3}\right)^{-1} \cdot s_{H_{2}}\left(a_{3}\right) \cdot s_{H_{1}}(e)+\epsilon \cdot s_{H_{2}}(e)+s_{H_{3}}\left(a_{1}\right)^{-1} \cdot s_{H_{2}}\left(a_{1}\right) \cdot s_{H_{3}}(e) \in K_{0} .
$$

Proof. For $e \in L$, the assertion is clear in view of $s_{H_{i}}(e)=0$ for $1 \leq i \leq 3$.

For $e=a_{1}$, the assertion means:

$$
\epsilon \cdot s_{H_{2}}\left(a_{1}\right)+s_{H_{2}}\left(a_{1}\right) \in K_{0}
$$

this is trivial.

Similarly, the assertion follows for $e=a_{3}$.

For $e=a_{2}$, the assertion is:

$$
s_{H_{1}}\left(a_{3}\right)^{-1} \cdot s_{H_{2}}\left(a_{3}\right) \cdot s_{H_{1}}\left(a_{2}\right)+s_{H_{3}}\left(a_{1}\right)^{-1} \cdot s_{H_{2}}\left(a_{1}\right) \cdot s_{H_{3}}\left(a_{2}\right) \in K_{0} .
$$

This holds by Lemma 5.4 ii) and Remark 2.6 ii).

In case $e \in\left(H_{1} \cup H_{2} \cup H_{3}\right) \backslash\left(L \cup\left\{a_{1}, a_{2}, a_{3}\right\}\right)$, the assertion follows from the facts just claimed and Lemma 5.4 iii).

Finally, assume that $e=: a_{4} \in E \backslash\left(H_{1} \cup H_{2} \cup H_{3}\right)$, and let $H_{4}$ denote the uniquely determined hyperplane in $M$ containing $L \cup\left\{a_{4}\right\}$; more explicitly, one has $H_{4}=\sigma\left(L \cup\left\{a_{4}\right\}\right)$, where $\sigma$ denotes the closure operator of $M$.

By applying Lemma $5.4 \mathrm{ii}$ ) to the three hyperplanes $H_{1}, H_{2}, H_{4}$ or $H_{1}, H_{3}, H_{4}$, respectively, we get:

$$
\begin{aligned}
& s_{H_{4}}\left(a_{2}\right) \cdot s_{H_{4}}\left(a_{1}\right)^{-1}=\epsilon \cdot s_{H_{1}}\left(a_{2}\right) \cdot s_{H_{1}}\left(a_{4}\right)^{-1} \cdot s_{H_{2}}\left(a_{4}\right) \cdot s_{H_{2}}\left(a_{1}\right)^{-1}, \\
& s_{H_{4}}\left(a_{3}\right) \cdot s_{H_{4}}\left(a_{1}\right)^{-1}=\epsilon \cdot s_{H_{1}}\left(a_{3}\right) \cdot s_{H_{1}}\left(a_{4}\right)^{-1} \cdot s_{H_{3}}\left(a_{4}\right) \cdot s_{H_{3}}\left(a_{1}\right)^{-1} .
\end{aligned}
$$

Furthermore, Remark 5.1 yields for the circuit $C$ and the hyperplane $H_{4}$ :

$$
f_{C}\left(a_{1}\right) \cdot s_{H_{4}}\left(a_{1}\right)+f_{C}\left(a_{2}\right) \cdot s_{H_{4}}\left(a_{2}\right)+f_{C}\left(a_{3}\right) \cdot s_{H_{4}}\left(a_{3}\right) \in K_{0} .
$$

Together with the relations

$$
\begin{aligned}
& f_{C}\left(a_{2}\right) \cdot f_{C}\left(a_{1}\right)^{-1}=\epsilon \cdot s_{H_{3}}\left(a_{1}\right) \cdot s_{H_{3}}\left(a_{2}\right)^{-1}, \\
& f_{C}\left(a_{3}\right) \cdot f_{C}\left(a_{1}\right)^{-1}=\epsilon \cdot s_{H_{2}}\left(a_{1}\right) \cdot s_{H_{2}}\left(a_{3}\right)^{-1},
\end{aligned}
$$

which hold by Lemma 5.2 iii), we get:

$$
s_{H_{4}}\left(a_{1}\right)+\epsilon \cdot s_{H_{3}}\left(a_{1}\right) \cdot s_{H_{3}}\left(a_{2}\right)^{-1} \cdot s_{H_{4}}\left(a_{2}\right)+\epsilon \cdot s_{H_{2}}\left(a_{1}\right) \cdot s_{H_{2}}\left(a_{3}\right)^{-1} \cdot s_{H_{4}}\left(a_{3}\right) \in K_{0} .
$$

After multiplying with $s_{H_{4}}\left(a_{1}\right)^{-1}$, we get, together with the above formulas:

$$
\begin{gathered}
1+s_{H_{1}}\left(a_{2}\right) \cdot s_{H_{1}}\left(a_{4}\right)^{-1} \cdot s_{H_{2}}\left(a_{4}\right) \cdot s_{H_{2}}\left(a_{1}\right)^{-1} \cdot s_{H_{3}}\left(a_{1}\right) \cdot s_{H_{3}}\left(a_{2}\right)^{-1} \\
+s_{H_{1}}\left(a_{3}\right) \cdot s_{H_{1}}\left(a_{4}\right)^{-1} \cdot s_{H_{3}}\left(a_{4}\right) \cdot s_{H_{3}}\left(a_{1}\right)^{-1} \cdot s_{H_{2}}\left(a_{1}\right) \cdot s_{H_{2}}\left(a_{3}\right)^{-1} \in K_{0} .
\end{gathered}
$$


Together with Lemma 5.4 ii), we get:

$$
\begin{gathered}
1+\epsilon \cdot s_{H_{1}}\left(a_{3}\right) \cdot s_{H_{2}}\left(a_{3}\right)^{-1} \cdot s_{H_{2}}\left(a_{4}\right) \cdot s_{H_{1}}\left(a_{4}\right)^{-1} \\
+s_{H_{1}}\left(a_{3}\right) \cdot s_{H_{2}}\left(a_{3}\right)^{-1} \cdot s_{H_{2}}\left(a_{1}\right) \cdot s_{H_{3}}\left(a_{1}\right)^{-1} \cdot s_{H_{3}}\left(a_{4}\right) \cdot s_{H_{1}}\left(a_{4}\right)^{-1} \in K_{0} .
\end{gathered}
$$

Multiplying with the product $s_{H_{1}}\left(a_{4}\right) \cdot s_{H_{1}}\left(a_{3}\right)^{-1} \cdot s_{H_{2}}\left(a_{3}\right)$ yields the assertion for $e=a_{4}$.

REMARK 5.6. In [7] and [8], we have examined the Tutte group of a matroid and proved already results closely related to Proposition 5.5 for matroids with coefficients of finite rank by using Tutte groups. In forthcoming papers, the concept of the Tutte group will be extended to matroids of arbitrary rank.

DEFINITION 5.7. A dual pair of oriented matroids is a dual pair of matroids with coefficients in the fuzzy ring $K=\mathbb{R} / / \mathbb{R}^{+}$. If $M$ - and $M^{*}$ - are the corresponding underlying matroids, then $M$, together with the corresponding family $\left(f_{C}\right)_{C \in C}$ of circuit functions - or $M^{*}$, together with the corresponding family $\left(r_{D}\right)_{D \in \mathcal{C}^{*}}$, respectively, is called an oriented matroid.

As above, we consider again hyperplane functions $s_{H}$ for hyperplanes $H$ in the given oriented matroid - where $E \backslash H \in \mathcal{C}^{*}$.

REMARK 5.8. Assume that $M$ is an arbitrary oriented matroid defined on $E$ with $\left(s_{H}\right)_{H \in \mathcal{H}}$ as its family of hyperplane functions. With $1=\mathbb{R}^{+}$and $\epsilon=\mathbb{R}^{-}$, we write for $H \in \mathcal{H}$ :

$$
H^{+}=s_{H}^{-1}(\{1\}), \quad H^{-}=s_{H}^{-1}(\{\epsilon\}) .
$$

Certainly, $E$ is the disjoint union of $H, H^{+}$and $H^{-}$.

$H^{+}$and $H^{-}$are called the halfspaces of the hyperplane $H$.

DEFINITION 5.9. Suppose that $H_{1}, H_{2}$ are hyperplanes in the given matroid $M$. Then the pair $\left(H_{1}, H_{2}\right)$ is called a modular pair of hyperplanes, if $\mathrm{H}_{1} \cap \mathrm{H}_{2}$ is a hyperline.

In this case, the pair $\left(E \backslash H_{1}, E \backslash H_{2}\right)$ is also called a modular pair of cocircuits - in $M$, while it is, of course, a modular pair of circuits in $M^{*}$.

PROPOSITION 5.10. Suppose that $\left(H_{1}, H_{2}\right)$ is a modular pair of hyperplanes in an oriented matroid $M$ defined on $E$. Furthermore, assume that

$$
a \in\left({H_{1}}^{+} \cap{H_{2}}^{-}\right) \cup\left({H_{1}}^{-} \cap H_{2}^{+}\right) .
$$

Then, the uniquely determined hyperplane $H_{3}$ containing $\left(H_{1} \cap H_{2}\right) \cup\{a\}$ satisfies

$$
\begin{gathered}
\text { either } \mathrm{H}_{3}{ }^{+} \subseteq \mathrm{H}_{1}{ }^{+} \cup \mathrm{H}_{2}{ }^{+} \text {and } \mathrm{H}_{3}{ }^{-} \subseteq \mathrm{H}_{1}{ }^{-} \cup \mathrm{H}_{2}{ }^{-} \\
\quad \text { or } \mathrm{H}_{3}{ }^{+} \subseteq \mathrm{H}_{1}{ }^{-} \cup \mathrm{H}_{2}{ }^{-} \text {and } \mathrm{H}_{3}{ }^{-} \subseteq{\mathrm{H}_{1}}^{+} \cup \mathrm{H}_{2}{ }^{+} .
\end{gathered}
$$

Proof. By Proposition 5.5, there exist $\alpha_{1}, \alpha_{2}, \alpha_{3} \in\{1, \epsilon\}$ such that for all $e \in E$ we have:

$$
\alpha_{1} \cdot s_{H_{1}}(e)+\alpha_{2} \cdot s_{H_{2}}(e)+\alpha_{3} \cdot s_{H_{3}}(e) \in K_{0} .
$$

Note that $s_{H_{3}}(a)=0$ and that - by assumption: $s_{H_{1}}(a)=\epsilon \cdot s_{H_{2}}(a)$.

By putting $e=a$, we obtain: $\alpha_{1}=\alpha_{2}$.

If $\alpha_{3}=\epsilon \cdot \alpha_{1}=\epsilon \cdot \alpha_{2}$, we get for all $e \in{H_{3}}^{+}$:

$$
e \in \mathrm{H}_{1}^{+} \text {or } \quad e \in \mathrm{H}_{2}^{+} \text {. }
$$

Analogously, we get in this case: $\mathrm{H}_{3}{ }^{-} \subseteq \mathrm{H}_{1}{ }^{-} \cup \mathrm{H}_{2}{ }^{-}$.

If, otherwise, one has $\alpha_{1}=\alpha_{2}=\alpha_{3}$, we get:

$$
\mathrm{H}_{3}{ }^{+} \subseteq{\mathrm{H}_{1}}^{-} \cup \mathrm{H}_{2}{ }^{-} \text {and } \mathrm{H}_{3}{ }^{-} \subseteq \mathrm{H}_{1}{ }^{+} \cup \mathrm{H}_{2}{ }^{+} \text {. }
$$

REMARK 5.11. Within the classical theory of finite oriented matroids, it is often required that all circuits $C_{1}, C_{2}$ satisfy the following condition: 
Whenever $a \in\left(C_{1}{ }^{+} \cap C_{2}{ }^{-}\right) \cup\left(C_{1}{ }^{-} \cap C_{2}{ }^{+}\right)$, then there exists a circuit $C_{3}$ with $a \notin C_{3}$ as well as:

$$
C_{3}{ }^{+} \subseteq{C_{1}}^{+} \cup{C_{2}}^{+} \text {and } C_{3}^{-} \subseteq{C_{1}}^{-} \cup C_{2}^{-} \text {. }
$$

By dualizing this condition, one gets the elimination property as stated in Proposition 5.10 - for all pairs of different hyperplanes and not only for modular pairs, if $E$ is finite. However, for infinite $E$, the condition that $\left(H_{1}, H_{2}\right)$ is a modular pair is essential:

Consider, for example, the matroid $\bar{M}$ defined on $\mathbb{R}^{2}$ given by affine independence, put $E:=$ $\{(x, y) \mid x, y \in \mathbb{R}, x \neq-y\} \cup\{(-1,1)\}$ and $M:=\bar{M} \mid E$. Moreover, put

$$
\begin{array}{rlrl}
H_{1} & :=\left\{(x, 0) \mid x \in \mathbb{R}^{*}\right\}, & H_{2}:=\left\{(0, y) \mid y \in \mathbb{R}^{*}\right\}, \\
H_{1}{ }^{+}:=\{(x, y) \in E \mid y>0\}, & H_{1}^{-}:=\{(x, y) \in E \mid y<0\}, \\
H_{2}{ }^{+}:=\{(x, y) \in E \mid x>0\}, & H_{2}^{-}:=\{(x, y) \in E \mid x<0\},
\end{array}
$$

as well as $a:=(-1,1)$. Then one has $a \in H_{1}{ }^{+} \cap H_{2}{ }^{-}$. However, every hyperplane $H$ in $M$ that passes through $a$ partitions $E \backslash H$ into 2 halfspaces such that (exactly) one of these intersects all 4 sets $\mathrm{H}_{1}{ }^{+} \cap \mathrm{H}_{2}{ }^{+}, \mathrm{H}_{1}{ }^{+} \cap \mathrm{H}_{2}{ }^{-}, \mathrm{H}_{1}{ }^{-} \cap \mathrm{H}_{2}{ }^{-}, \mathrm{H}_{1}{ }^{-} \cap \mathrm{H}_{2}{ }^{+}$. Note that the set $\{(x, y) \in E \mid x=-y\}$ is not a hyperplane in $M$ but merely contains $a$. - However, note also that $H_{1} \cap H_{2}=\varnothing$, whence $\left(H_{1}, H_{2}\right)$ is not a modular pair of hyperplanes in $M$.

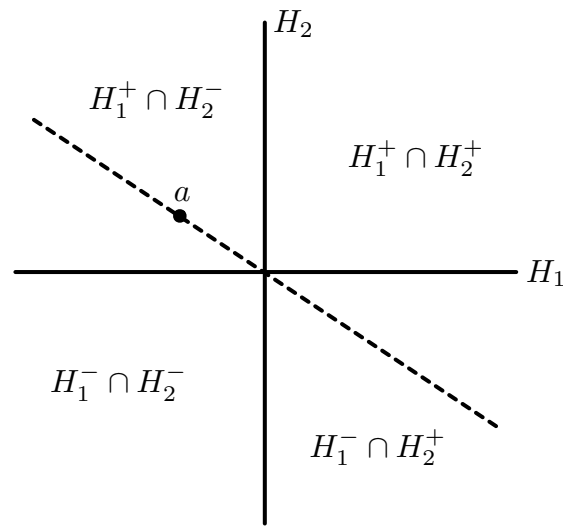

The next definition concerning weakly oriented matroids is slightly different - but, in the sense of [6] - combinatorially equivalent to that given in [12].

DEFINITION 5.12. A dual pair of weakly oriented matroids is a dual pair of matroids with coefficients in the fuzzy ring $K_{w}:=\mathbb{F}_{7} / /\left(\mathbb{F}_{7}^{*}\right)^{2}$.

REMARK 5.13. In what follows, put $U_{7}:=\left(\mathbb{F}_{7}^{*}\right)^{2}=\left\{x^{2} \mid x \in \mathbb{F}_{7}^{*}\right\}$.

Moreover, write

$$
0=\{0\}, \quad 1=U_{7}, \quad \epsilon=-U_{7}, \quad q=\mathbb{F}_{7}^{*}, \quad \omega=\mathbb{F}_{7} .
$$

Then the fuzzy ring $K_{w}$ satisfies:

$$
K_{w}=\{0,1, \epsilon, q, \omega\}, \quad K_{0}=\{0, \omega\}, \quad K_{w}{ }^{*}=\{1, \epsilon\} .
$$

Note that $K_{w}$ is a finitary fuzzy ring by Proposition 3.6. 
Furthermore, we have the following addition and multiplication tables:

\begin{tabular}{c|ccccc}
+ & 0 & 1 & $\epsilon$ & $q$ & $\omega$ \\
\hline 0 & 0 & 1 & $\epsilon$ & $q$ & $\omega$ \\
1 & 1 & $q$ & $\omega$ & $\omega$ & $\omega$ \\
$\epsilon$ & $\epsilon$ & $\omega$ & $q$ & $\omega$ & $\omega$ \\
$q$ & $q$ & $\omega$ & $\omega$ & $\omega$ & $\omega$ \\
$\omega$ & $\omega$ & $\omega$ & $\omega$ & $\omega$ & $\omega$
\end{tabular}

\begin{tabular}{c|ccccc}
$\cdot$ & 0 & 1 & $\epsilon$ & $q$ & $\omega$ \\
\hline 0 & 0 & 0 & 0 & 0 & 0 \\
1 & 0 & 1 & $\epsilon$ & $q$ & $\omega$ \\
$\epsilon$ & 0 & $\epsilon$ & 1 & $q$ & $\omega$ \\
$q$ & 0 & $q$ & $q$ & $q$ & $\omega$ \\
$\omega$ & 0 & $\omega$ & $\omega$ & $\omega$ & $\omega$
\end{tabular}

Particularly, any three units $u_{1}, u_{2}, u_{3} \in K^{*}$ satisfy:

$$
u_{1}+u_{2}+u_{3}=\omega
$$

Moreover, we can carry over the notations $\mathrm{H}^{+}, \mathrm{H}^{-}$for halfspaces of hyperplanes in oriented matroids to weakly oriented matroids.

We conclude:

Two families $\left(f_{C}\right)_{C \in C}$ of circuit functions and $\left(s_{H}\right)_{H \in \mathcal{H}}$ of hyperplane functions, respectively, automatically constitute a dual pair of matroids with coefficients in $K_{w}$, if (DPi) holds and (DPii) holds (at least) for all circuits $C$ and hyperplanes $H$ with $|C \backslash H|=2$.

Finally, note the following:

In [12], the product $q \cdot q$ takes the value $\omega$ instead of $q$; elsewhere, the values in the established tables coincide. The structure studied by M. Wagowski is not a quotient of a field and a subgroup of its group of units, but has the advantage that it is a semiring; that means, it satisfies the distributivity law. However, distributivity is not necessary for our purposes.

Similarly to Proposition 5.10, one has:

PROPOSITION 5.14. Suppose that $\left(H_{1}, H_{2}\right)$ is a modular pair of hyperplanes in a weakly oriented matroid $M$ defined on $E$. Furthermore, assume that

$$
a \in\left(H_{1}{ }^{+} \cap H_{2}^{-}\right) \cup\left(H_{1}^{-} \cap H_{2}^{+}\right) .
$$

Then, the uniquely determined hyperplane $H_{3}$ with $\left(H_{1} \cap H_{2}\right) \cup\{a\} \subseteq H_{3}$ satisfies either

$$
\mathrm{H}_{3}{ }^{+} \cap H_{i} \subseteq{H_{j}}^{+} \text {and } H_{3}{ }^{-} \cap H_{i} \subseteq H_{j}^{-} \text {for }\{i, j\}=\{1,2\}
$$

or

$$
\mathrm{H}_{3}{ }^{+} \cap \mathrm{H}_{i} \subseteq \mathrm{H}_{j}^{-} \text {and } \mathrm{H}_{3}{ }^{-} \cap H_{i} \subseteq H_{j}{ }^{+} \text {for }\{i, j\}=\{1,2\} \text {. }
$$

Note that Proposition 5.10 and Proposition 5.14 show directly that every dual pair of oriented matroids is also a dual pair of weakly oriented matroids.

For valuated matroids, we clearly have the following proposition - based on Example 3.4 and the tables presented there:

PROPOSITION 5.15. Assume that $\Gamma$ is a linearly ordered abelian group. Two families $\left(f_{C}\right)_{C \in C}$ and $\left(s_{H}\right)_{H \in \mathcal{H}}$ of circuit functions $f_{C}: E \rightarrow \Gamma \cup\{0\}$ and hyperplane functions $s_{H}: E \rightarrow \Gamma \cup\{0\}$, respectively, define a dual pair of valuated matroids, if, beyond Axiom (DPi), the following condition holds:

Given a circuit $C$ and a hyperplane $H$ with $C \backslash H \neq \varnothing$, there exist two different elements $e_{1}, e_{2} \in C \backslash H$ such that for all $a \in C \backslash H$ one has:

$$
f_{C}(a) \cdot s_{H}(a) \leq f_{C}\left(e_{1}\right) \cdot s_{H}\left(e_{1}\right)=f_{C}\left(e_{2}\right) \cdot s_{H}\left(e_{2}\right) .
$$

REMARK 5.16. For tame matroids, the condition in Proposition 5.15 is equivalent to the following one:

Given a circuit $C$, a hyperplane $H$ with $C \backslash H \neq \varnothing$ as well as an element $a \in C \backslash H$, there exists an element $e \in C \backslash H$ such that

$$
f_{C}(a) \cdot s_{H}(a) \leq f_{C}(e) \cdot s_{H}(e)
$$

Namely, if $C \backslash H$ is finite, both conditions mean that a maximum value of all products of the shape $f_{C}(e) \cdot s_{H}(e)$ for $e \in C \backslash H$ exists at all - and is attained at least twice. If, however, $C \backslash H$ is infinite, the last condition is fulfilled also if no maximum value among the products considered exists. Hence,

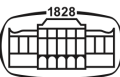


for wild matroids, the condition stated here is more general than that stated in Proposition 5.15. But, as pointed out, this proposition is tailored to matroids with coefficients in the fuzzy ring $K_{\Gamma}$.

It seems an interesting question whether it makes also sense to study more general vauated matroids within the scope of this remark.

\section{ACKNOWLEDGEMENTS}

The author thanks R. Diestel, N. Bowler, F. Kalhoff, M. Hoya, S. Kaspar, and N. Ay for several discussions concerning the main ideas developed in this paper.

\section{REFERENCES}

[1] Bland, R. G., And Vergnas, M. L. Orientability of matroids. F. Combin. Theory Ser. B 24 (1978), 94-123.

[2] Borujeni, H. A., And Bowler, N. Thin sums matroids and duality. Adv. Math. 271 (2015), 1-29.

[3] Bowler, N., ANd Carmesin, J. Matroids with an infinite circuit-cocircuit intersection. $\mathcal{F}$. Combin. Theory Ser. B 107 (2014), 78-91.

[4] Bruhn, H., Diestel, R., Kriesell, M., Pendavingh, R., And Wollan, P. Axioms for infinite matroids. Adv. Math. 239 (2013), 18-46.

[5] Dress, A. W. M. Chirotopes and oriented matroids. Bayreuth. Math. Schr. 21 (1985), 14-68. Tagungsbericht 2. Sommerschule Diskrete Strukturen Bayreuth.

[6] Dress, A. W. M. Duality theory for finite and infinite matroids with coefficients. Adv. Math. 59 (1986), 97-123.

[7] Dress, A. W. M., And Wenzel, W. Geometric algebra for combinatorial geometries. Adv. Math. 77 (1989), 1-36.

[8] Dress, A. W. M., AND Wenzel, W. Grassmann-plücker relations and matroids with coefficients. Adv. Math. 86 (1991), 68-110.

[9] Dress, A. W. M., And Wenzel, W. Valuated matroids. Adv. Math. 93 (1992), 214- 250.

[10] Gutierrez NovoA, L. On $n$-ordered sets and order completeness. Pacific f. Math. 15 (1965), 1337-1345.

[11] Oxley, J. Matroid Theory. Oxford University Press, Oxford, 1992.

[12] Wagowski, M. Matroid signatures coordinatizable over a semiring. European f. Combin. 10 (1989), 393-398.

Open Access statement. This is an open-access article distributed under the terms of the Creative Commons AttributionNonCommercial 4.0 International License (https://creativecommons.org/licenses/by-nc/4.0/), which permits unrestricted use, distribution, and reproduction in any medium for non-commercial purposes, provided the original author and source are credited, a link to the CC License is provided, and changes - if any - are indicated. 